Project 90171

\title{
Phosphate Barriers for Immobilization of Uranium Plumes
}

\author{
Burns, Peter C.
}

University of Notre Dame

RESULTS TO DATE: Project Title: Phosphate Barriers for Immobilization of Uranium Plumes

Lead Principal Investigator: Johnathan P. Icenhower, Pacific Northwest National Laboratory

Co-Principal Investigator: Peter C. Burns, Department of Civil Engineering and Geological Sciences, University of Notre Dame, Notre Dame, IN 46556, (574) 631-5380, pburns@nd.edu

Number of Graduate Students Involved: 2

Number of Undergraduate Students Involved: 2

Uranium contamination of the subsurface has remained a persistent problem plaguing remedial design at sites across the U.S. that were involved with production, handling, storage, milling, and reprocessing of fissile uranium for both civilian and defense related purposes. Remediation efforts to date have relied upon excavation, pump-and-treat, or passive remediation barriers (PRB?s) to remove or attenuate uranium mobility. Documented cases convincingly demonstrate that excavation and pump-and-treat methods are ineffective for a number of highly contaminated sites. There is growing concern that use of conventional PRB?s, such as zero-valent iron, are a temporary solution to a problem that will persist for thousands of years. Alternatives to the standard treatment methods are therefore warranted. The core objective of our research is to demonstrate that a phosphorous amendment strategy will result in a reduction of dissolved uranium to below the proposed drinking water standard. Our hypothesis is that long-chain polyphosphate compounds forestall precipitation of sparingly soluble uranyl phosphate compounds, which is key to preventing fouling of wells at the point of injection. Our other fundamental objective is to synthesize and correctly characterize the uranyl phosphate phases that form in the geochemical conditions under consideration. This report summarizes work conducted at the University of Notre Dame through November of 2003 under DOE grant DE-FG07-02ER63489, which has been funded since September, 2002. The objectives at Notre Dame are development of synthesis techniques for uranyl phosphate phases, together with detailed structural and chemical characterization of the myriad of uranyl phosphate phases that may form under geochemical conditions under consideration.

We have developed novel synthesis techniques for many of the uranyl phosphates of interest. Superb single crystals have been obtained by diffusion of crystal nutrients into silica-based gels, as this approach retards crystal nucleation and enhances growth of a small number of crystals. We have also developed mild hydrothermal techniques that provide high-purity uranyl phosphate compounds. Using a CCD-based singlecrystal diffractometer, full structure and chemical characterizations have been completed for 21 uranyl phosphates. We emphasize that many of these materials were poorly understood at the outset of our research, with unknown or partially known structures, and uncertainties concerning the water content of the phases. This component of the overall research program is providing a much improved understanding of the synthesis and structures of the uranyl phosphate group, which is an essential step prior to measuring solubilities of these phases. Our development of new synthesis techniques for uranyl phosphates is a necessary step towards measuring the solubilities of well-characterized and pure materials. 
